\title{
Congruent Facilitation of Simulations and Games
}

\author{
Elyssebeth Leigh ${ }^{1}$ and Laraine Spindler ${ }^{2}$
}

In a review of the journal Simulations and Gaming (Sage Publications) we found less than $10 \%$ of all articles made reference to requirements of the person/s facilitating a simulation or game. It seemed that many writers did not regard facilitation as sufficiently important, or were unaware that its nature can be problematic.

Until events necessitated critical re-analysis of our practice, we were similarly unconcerned about our own facilitation skills. Once we began examining facilitation processes, new insights into the facilitation role emerged. These insights especially concern the way in which personal preferences appear to have a major influence on choices and behaviours when facilitating experiential learning activities. Two sets of choices emerge as particularly relevant. The first concerns choices about the type of simulation or game chosen; the second concerns the preferred facilitation style and observable behaviours. We first wrote about these in 1998 (Leigh and Spindler 1998) and have continued our explorations in subsequent papers (Leigh 2003a, Leigh 2003b, Leigh and Spindler - in press).

In this chapter we briefly describe our earlier work, and extend the proposition that personal teaching and learning philosophies often have a greater influence on choices and actions than requirements of specific educational outcomes. For example, given similar learning outcomes, someone who sees learning as a highly structured process requiring tight control is likely to choose a quite different approach to someone who regards learning as an emergent process dependent on interactions among learner, processes and content.

ISAGA 2003 we used a collaborative research strategy to explore these propositions. We had developed instruments to assist in identifying philosophical stances, preferences for simulations and games formats, and facilitation practices. During the workshop participants were able to use these instruments to identify personal patterns among these factors. There was sufficient support for our propositions to encourage further research.

\section{Defining simulations and games}

When discussing definitions and types of simulations in use around the world it is easy to see that the choices are immensely varied. What 'are' and 'are not' simulations, how to manage, design, learn from and behave in simulations - are all subject to debate. While preparing this paper one of us was invited to complete two electronic surveys about the field. Neither site provided a definition of 'simulation', both apparently assuming that anyone completing the survey shared

\footnotetext{
${ }^{1}$ Faculty of Education, University of Technology, Sydney

${ }^{2}$ Institute for International Studies, University of Technology, Sydney
} 
their (un-stated) assumptions about what the term means. One was for a technology based Australian simulation association and the other for an international technology teaching and research centre. The assumption, that there is no problem about the 'meaning' of the term, emphasises the need to reduce the chance of misunderstanding by providing our working definition -

Simulations and games include all interactive representations of perceived reality past, present, future - used for learning purposes (Leigh 2003)

Such a broad definition allows consideration of the widest possible spectrum of activities and we encourage readers to think about their own definition/s, and to regularly review their personal schemas for the field. To pursue our exploration of the facilitator's role and choices we use three arrangements from a broad range of possible models for categorising simulations. We are aware that other equally useful arrangements exist and intend to include consideration of them in future work.

\section{A 'Spectrum' approach}

Taylor (1977)used a 'spectrum' approach to explain to educators of town planners the potential of simulations and games as teaching media. He arranged them from 'most' to 'least' real as models of human activity. He considered case studies to be 'most real' and electronic simulators to be 'least real' based on how materials mediate learning. Case studies are almost 'real' with little distance between player and 'reality' while mechanical simulators interpose extensive technology-based mediating elements between players and 'reality'. While Taylor's spectrum is nearly thirty years old and computer-based simulators now provide near 'perfect' representations of $e . g$. flying a plane, his spectrum still emphasises the importance of taking into account the mediating role of technology and materials.

\section{A 'Relational' Approach}

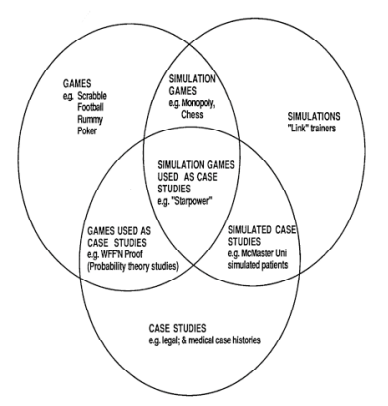

A useful model arranges simulations, games and case studies as three elements in a Venn diagram, creating seven formats (Ellington, 1999)

1. Pure games

2. Games used as simulations

3. Pure simulations

4. Pure case studies

5. Simulations used as case studies

6. Case studies used as games

7. Simulation games used as case studies

Figure 1 Ellington et al's relational approach to arranging simulations and games

Their arrangement is especially useful for choosing activities for learning contexts. For example a 'game' has rules and time constraints but need not be like reality, while a 'simulation' must specifically represent an aspect of reality (or potential reality), and a 'case study' must enable in-depth analysis of content. 


\section{A 'Drama' perspective}

Although useful in understanding materials and rules etc. neither of these explain how to manage the learning process. To do this requires something different. We have adopted Christopher and Smith's categorising of activities as 'open' or 'closed' (Christopher and Smith 1987) drawing on concepts from theatrical drama to distinguish between two quite different types of games.

'Closed' games, rely on the facilitator for control and authority to arrive at predetermined learning points. While the route to solutions may vary, the overall action and debriefing processes do not. In contrast, 'open' games inhibit the facilitator from being someone who 'knows the desired answer'. They structure the role to minimise a facilitator's capacity to help participants arrive at 'the right place' since there is no 'one right place'. Participants a journey, as they encounter moments of insight, while initiating events and experiencing emotions that may direct their attention in any number of likely, or unanticipated, directions. The facilitator supports and guides, reassures and encourages - does not direct, and never 'teaches' the meaning of what is happening.

\section{Assumptions about facilitation}

Both 'closed' and 'open' forms of simulation or game can contribute to rich learning experiences. But each makes quite different assumptions about how learning occurs and how participants and facilitators are to behave. A 'closed' game assumes that participants need guidance and the facilitator is there to provide it. An 'open' game assumes that participants create the experience they need to have, in order to learn. It further assumes that dis-order and confusion are likely to emerge as part of this process, and that this provides a 'container' within which 'deep learning' is likely to occur. The facilitator must not disturb the emergence of such disorderly 'chaos' but can offer support as participants travel forward to the destination being created by their actions.

We propose that facilitators who regard knowledge as an 'object' to be possessed, acquired as 'facts', and 'contained' in words will prefer the more structured form of 'closed' games. Conversely, facilitators who regard knowledge as emerging from the process, acquired through both emotional responses and examination of facts and 'things', will prefer the sense of 'journey' allowed in 'open' simulations and the way that the unexpected and unanticipated become vital forces for understanding that go beyond acquisition of data and facts.

As we explored these perspectives we developed the concept of the 'vigilant observer' - see Figure 1 below - and identified two factors linked to developing the capability for using 'open' games. We saw these as being

a) the degree of emotional detachment a facilitator brings to the setting, and

b) acceptance of the possibility of an unidentified number of possible goals, as a legitimate notion when designing and/or managing the process 


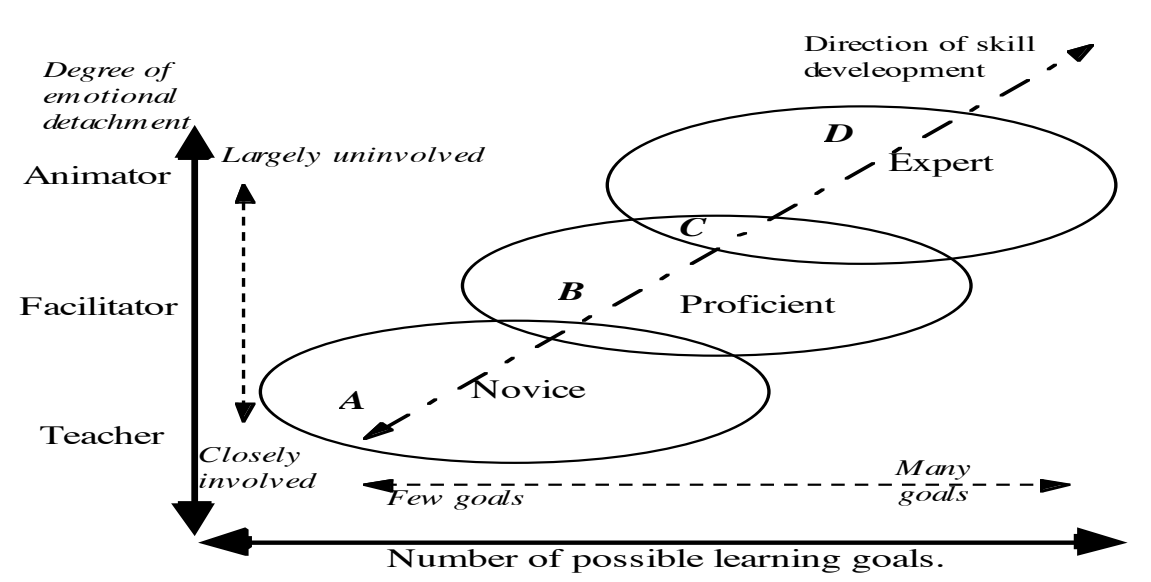

Figure 2 Emotional detachment and learning goals - linking 'novice', 'proficient' and 'expert' presenters of simulations

We used this to develop a spectrum with end points of 'moderator' and 'improviser' signalling the respective attitudes to the task of managing the learning. The 'improviser' is more comfortable with 'open' simulations and has similar characteristics to the 'animator' described by Boud et al (Boud and Miller 1996) with an emphasis on emotional detachment and acceptance of ambiguity. A moderator prefers 'closed' simulations and regards their task as being to ensure achievement of concrete intentions. The improviser works with what emerges from the action, being able to improvise from moment to moment, seeing the goal as assisting individuals to attend to their own experiences rather than directing attention to designated topics. Table 1 illustrates differences between 'moderators' and 'improvisers' emerging as the outcome of personal decisions made (often tacitly) about such things as: the relative importance of content knowledge; the need to control the action; the power relationships between 'learner' and 'teacher' roles; and beliefs about what constitutes 'appropriate' methodologies.

\begin{tabular}{|l|}
\hline Moderators of learning contexts \\
1. Aim to understand participant activity \\
and group dynamics in order to control \\
behaviour. \\
2. In their contexts the structure of learn- \\
ing is stable clear self-evident. \\
3. A Moderator's basic assumption is that \\
learning requires \\
- Orderliness and attention to detail \\
- Stability, with the teacher in control \\
- emotional constraint (except for fear)
\end{tabular}

Improvisers in learning contexts

1. Aim to understand the interactions and flow /patterns of the action in order to be ready to support and challenge the continuum of learning.

2. For them the patterns of learning emerge, are explored and are supported.

3. An Improviser's basic assumption is that learning creates:

- turbulence (emotional, and physical)

- discovery

- challenges to beliefs, and practice

Table 1: Educators as supporters of learning - a comparative arrangement of behaviours 
Our concern is that novice facilitators, unable to differentiate between 'closed' and 'open' forms may make errors in their management of the learning including:

- Stretching 'closed' simulations beyond their design parameters, by treating them as 'open' and therefore

- encourage participants to 'unpack' ideas that are not fully within the scope of the game but appear 'interesting'

- claim that an activity provides more variety than it can sustain upon inspection

- expect a game to operate as 'open' and telling participants how to make it so

- Limiting the potential of 'open' simulations by treating them as 'closed' and therefore

- require specific outcomes to be achieved

- $\quad$ ignore emergent learning, in favour of pre-set expectations

- contain action in narrower constraints than the designer's intentions

- ' 'rescue' learners temporarily lost in a 'morass' of potentials

- fail to encourage exploration of new potentials being created

We are more interested in errors related to treating 'open' games as if they are 'closed' - for two main reasons. The first is that such errors can generate a lot of emotional turmoil for both participants and facilitator if anyone begins to develop unrealistic or unattainable expectations about the others' role and behaviours. The second is that any potential for learning may be lost if participants and facilitator become seduced by the emotional turmoil and are unable to identify what learning is actually available (and indeed occurring).

To better understand problems in managing 'open' simulations, and to develop a means of improving the learning from our own experiences, we began to consider how teaching and learning preferences are shaped by educational philosophies and influence facilitation choices. A consideration of 'learning styles' and 'personality types' provides a brief introduction to thinking about how novice and experienced facilitators may better appreciate the implications of their choices in regard to games formats through better understanding their own profiles.

\section{Learning preferences}

David Kolb developed the concept of learning as occurring in a cycle, suggesting that adults prefer one or two of four steps in the cycle, but must use all four for new learning to be fully integrated (Kolb, Rubin et al. 1979). He suggested it is possible to map individual preferences in a way that enables anyone to understand more about their own 'beginning point' and become alert to the way this may shape their approach to learning activities. Honey and Mumford modified this approach by mapping the learning preferences in the form of a 'kite' (Honey and Mumford 1986). Their model suggests the following key characteristics of each of the four points of their 'kite: 
Activist - fully engages without bias in new experiences

Reflector- stands back to observe experiences from different perspectives

Theorist - adapts and integrates observations into complex logical theories

Pragmatist - tries out ideas, theories and techniques to see how they work in practice (Honey and Mumford 1986).

Figure 3 shows this approach, with the 'kite' of one of the authors superimposed on the gird as an example of what it can produce. This 'kite' suggests that its owner prefers action to 'kick start' new learning and has a pragmatic need for learning to be relevant to current practice. Observation and analysis remain subservi-

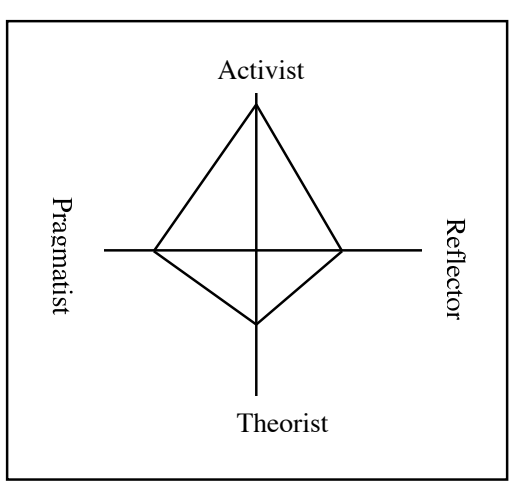

Figure 3 'Learning Styles' as a set of preferences (Honey \& Mumford,1986) ent to action. The kite's owner prefers to teach and learn via simulations and games, with a strong affinity for 'open' simulations as a challenging and rewarding way to help adult learners integrate present knowledge and new information.

A person with a preference for extensive reflection and careful analysis prior to action will be less likely to enjoy the 'free form' nature of 'open' simulations that require quick responses and allow little time for in-depth analysis of options. The comparative strengths of each one's preferences is likely to dictate responses to specific activities. Thus a knowledge of one's own learning preferences and its impact on the design and delivery of learning processes can assist facilitators to both extend their own repertoire of teaching strategies and be better prepared for the widest possible array of learners' responses.

\section{Personality 'types'}

In general terms personality theories aim to explain psychological aspects of human beings. A well known model influencing development of personality theory is that of Carl Jung (1974). He described four mental 'functions' and noted that

1) Everyone can perform all of them

2) Each function has an opposite; each pair of functions has an opposite pair

3) We prefer using some functions, and pairs, more than others

4) These habits can be generalized to describe "types" of people

We suggest that the S, T, J preferences prefer 'closed' simulations because of the opportunities they provide for clear, detailed analysis and development of logical, well structured arguments about specific learning outcomes. Conversely $\mathrm{N}, \mathrm{F}, \mathrm{P}$ preferences are more likely to choose 'open' simulations because of the prospects for exploring patterns, creating original action, building relationships and working with lots of information. 


\section{$\mathbf{S}$ and $\mathbf{N}$}

We take information in via our senses. When this Sensation Function (S) dominates perception, we prefer sensate things: certainty, precision, simplicity, practicality, concreteness. The Intuition (N) function perceives patterns and possibilities. An $\mathbf{N}$ sees objects as parts of patterns, implications, possibilities or theories. The $\mathbf{N}$ dreams, nothing is the same way twice; there must be change.

Until they understand these differences the two functions will not value each other. $\mathbf{S}$ calls $\mathbf{N}$ a lazy dreamer, and $\mathbf{N}$ considers $\mathbf{S}$ a plodder.

\section{$\mathbf{T}$ and $\mathbf{F}$}

As we take in information we use it via thinking and feeling. Thinking (T) analyses elements to reach an objective truth. Information is dealt with objectively, impersonally, logically. Thinkers make good planners because they lay events out in order. Feeling (F) types do not just have feelings; they use them to make value judgments to build relationships, compare things, act compassionately. T is irritated by $\mathbf{F}$ 's personalizing, who sees $\mathbf{T}$ as a cold fish.

\section{$\mathbf{P}$ and $\mathbf{J}$}

A Perceiving $(\mathbf{P})$ person takes in a lot of information before deciding and taking action. A Judging $(\mathbf{J})$ person takes in less information and decides more quickly. $\mathbf{P}$ may be slow; $\mathbf{J}$ may "shoot from the hip". (Putzel 2001)

Table 2: Brief summary of Myers Briggs Type Indicator Typology

We are cautious in our predictions, as we have only worked with limited data, however we received encouraging peer support at ISAGA 2003 and report on that aspect of the research in what follows.

In developing our approach we opted to work with a set of models that arrange key elements into orthogonal (right-angled) relationships creating four distinct stances in regard to each concept being examined. The three key models we chose to work with concern a) adult learning principles, b) project management types and a personality profile called 'Tetramap' which draws on a combination of 'western' and 'eastern' philosophies to help

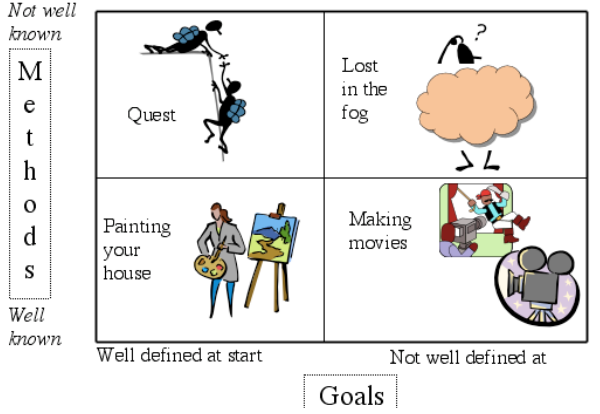

Figure 4 Turner's project types identify four distinct 'types' of approach to learning and being. To illustrate our own concepts in regard to facilitating the two forms of 'open' and 'closed' simulations and games we constructed a fourth model depicting the variety of approaches as related to a preference for focusing on 'individual' or 'group' learning. Each of the chosen models can be arranged such that the ends of the spectrum are not 'either/or' but 'both/and'. That is the factors can aligned as a 'more than/less than' equilibrium of both measures, rather than being regarded as 'possessing' or 'not possessing' each of the factors being considered in each model. For example Turner's four 'types of project' - shown in Figure 4- is based on the factors of a) knowledge of methods to be applied and b) tools to be used. Boud's 
conceptualisation of adult learning principles is at Figure 5 and our arrangement of the Tetramap elements is at Figure 6.

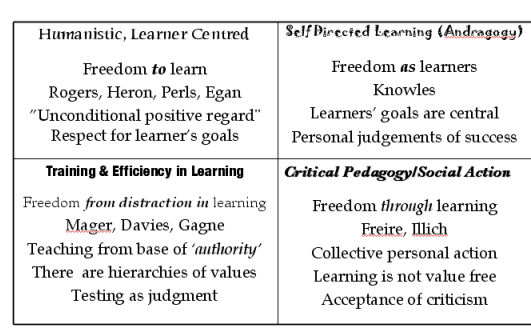

Figure 4 Four approaches to adult education (Boud 19**)

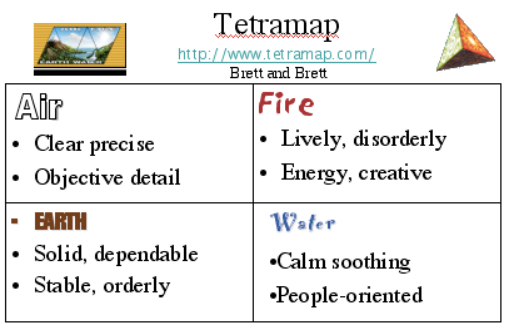

Figure 5 'Elements' based approach to personal styles

These models are all ones we use elsewhere in our adult education programs and each one provides an insight into a different aspect of the teaching and learning processes in which we are engaged.

\section{References}

Boud, D. and N. Miller, Eds. (1996). Working With Experience London, UK, Routledge Christopher, E. M. and L. E. Smith (1987). Leadership Training Through Gaming London Kogan Page

Duke, R. D. (1974). Gaming: the Futures Language. New York, USA, Halsted Press.

Ellington, H. (1999). Games and Simulations - Media for the New Millenium. 1999 SAGSET.

Honey, P. and A. Mumford (1986). The Manual Of Learning Styles. Maidenhead, UK Jung, C. (1974). Psychological Types. Princeton, NJ:, Princeton University Press.

Kolb, D. A., I. M. Rubin, et al. (1979). Organizational Psychology an experiential approach. Englewood Ciffs, USA, Prentice-Hall.

Leigh, E. (2003a) A Touchy Subject - People Factors In Simulations SimTECT 2003, Adelaide

Leigh, E. (2003b). What is expected of the facilitator of interactive learning? In Interactive Learning through Gaming and Simulation. H. G. Fred Percival, et al Edinburgh, ISAGA/SAGSET.

Leigh, E and Spindler, L (2003) Simulations and Games as Chaordic Learning Contexts (in press- Simulations \& Gaming Journal, Sage Publications)

Leigh, E. and L. Spindler (1998). 'Vigilant observer': Arole for facilitators of games/simulations. In Gaming/Simulation for Policy Development and Organizational Change. Jac Guerts, et al, Tilburg University Press

Putzel, R. (2001). XB Manual for A Learning Organization Colchester, VT, St. Michael's College.

Taylor, J. (1977). Instructional gaming procedures in planning education. In Aspects of Simulation and Gaming. J. Megarry. London, Kogan Page: 103 - 115.

Turner, R. 